\title{
Association between global leukocyte DNA methylation and cardiovascular risk in postmenopausal women
}

\author{
Ramon Bossardi Ramos ${ }^{1}$, Vitor Fabris ${ }^{1}$, Sheila Bunecker Lecke ${ }^{1,2}$, Maria Augusta Maturana ${ }^{1,4,5}$ \\ and Poli Mara Spritzer ${ }^{1,3^{*}}$
}

\begin{abstract}
Background: Genetic studies to date have not provided satisfactory evidence regarding risk polymorphisms for cardiovascular disease (CVD). Conversely, epigenetic mechanisms, including DNA methylation, seem to influence the risk of CVD and related conditions. Because postmenopausal women experience an increase in CVD, we set out to determine whether global DNA methylation was associated with cardiovascular risk in this population.

Methods: In this cross sectional study carried out in a university hospital, 90 postmenopausal women without prior CVD diagnosis (55.5 \pm 4.9 years, 5.8 [3.0-10.0] years since menopause) were enrolled. DNA was extracted from peripheral leukocytes and global DNA methylation levels were obtained with an ELISA kit. Cardiovascular risk was estimated by the Framingham General Cardiovascular Risk Score (10-year risk) (FRS). Clinical and laboratory variables were assessed. Patients were stratified into two CVD risk groups: low (FRS: $<10 \%, n=69$ ) and intermediate/high risk (FRS $\geq 10 \%, n=21$ ).
\end{abstract}

Results: Age, time since menopause, blood pressure, total cholesterol, and LDL-c levels were higher in FRS $\geq 10 \%$ group vs. FRS $<10 \%$ group. BMI, triglycerides, HDL-C, HOMA-IR, glucose and hsC-reactive protein levels were similar in the two groups. Global DNA methylation (\% 5mC) in the overall sample was $26.5 \%$ (23.6-36.9). The FRS $\geq 10 \%$ group presented lower global methylation levels compared with the FRS <10 \% group: $23.9 \%$ (20.6-29.1) vs. $28.8 \%$ (24.3-39.6), $p=0.02$. This analysis remained significant even after adjustment for time since menopause $(p=0.02)$.

Conclusions: Our results indicate that lower global DNA methylation is associated with higher cardiovascular risk in postmenopausal women.

Keywords: Epigenetics, 5-methylcytosine, Aging, Post-menopause, Cardiovascular diseases

\section{Background}

Epidemiologic data show that postmenopausal women experience an increase in cardiovascular disease (CVD), which could be associated with both aging and changes in hormonal status $[1,2]$. In this group, higher prevalence of subclinical CVD and cardiovascular (CV) risk

\footnotetext{
*Correspondence: spritzer@ufrgs.br

'Gynecological Endocrinology Unit, Division of Endocrinology, Hospital de Clínicas de Porto Alegre, Rua Ramiro Barcelos, 2350, Porto Alegre, RS 90035-003, Brazil

${ }^{3}$ Laboratory of Molecular Endocrinology, Department of Physiology, Universidade Federal do Rio Grande do Sul, Rua Ramiro Barcelos, 2350, Porto Alegre, RS 90035-003, Brazil

Full list of author information is available at the end of the article
}

factors has been associated with the decline in endogenous estrogen production [3-8].

Evidence indicates that CVD results from a complex interaction between environmental and genetic factors $[9,10]$. Thus, the formula proposed by the Framingham Heart study to calculate $\mathrm{CV}$ risk takes into account common behavioral, biochemical, and environmental aspects that may contribute to the development of CVD [11, 12]. In turn, genetic studies have shown limited predictive value of polymorphisms to explain CV risk factors [13]. This suggests that other factors might be influencing the estimated variability of CV risk $[14,15]$.

In a context in which traditional explanations are not sufficient to account for the link between disease, 
environment, and genetics, epigenetics emerges as a framework to provide insights into the bases of disease $[16,17]$. Epigenetics - the study of heritable changes in gene activity or function without changes in DNA sequence $[18,19]$ - is highly relevant to all disease processes, including CVD and its related conditions [20]. DNA methylation, a major epigenetic mechanism, is the process by which methyl groups are added to DNA, typically regulating tissue-specific gene expression, genomic imprinting, and X chromosome inactivation [21]. DNA methylation is established and maintained by a conserved family of DNA methyltransferases (DNMTs), and plays an important role in genome stability [22]. The importance of DNMTs is related to de novo DNA methyltransferases $3 \mathrm{a} / 3 \mathrm{~b}$ (DNMT3a/3b) synthesis, which will methylate unmethylated cytosines to induce differential patterns of methylation in the genome of early embryos. These patterns are subsequently copied from parental strands into daughter strands during DNA replication by maintenance DNMT1 [23].

Global DNA methylation may provide a broad picture of DNA methylation changes; previous studies have shown it to be one of the earliest molecular changes in the transition from a normal to a diseased cell [24, 25]. Besides, global DNA methylation has a high-throughput, is cost-effective, and provides quantitative results [26]. Global DNA methylation changes, including decreased global DNA methylation, have been associated with clinical and subclinical CVD risk components, such as atherosclerosis, hypertension, and coronary artery disease [26-30].

Therefore, the aim of the present study was to determine whether global DNA methylation is associated with $\mathrm{CV}$ risk in a sample of postmenopausal women with no evidence of clinical disease.

\section{Methods \\ Patients}

This cross-sectional study was carried out at the Gynecological Endocrinology Unit of Hospital de Clínicas de Porto Alegre, Brazil. Ninety postmenopausal women from a group of 97 participants described in a previous study [31] were included in the present analysis. Seven women from the original group were excluded because we were unable to detect a methylation signal in their serum samples. As previously described [31], inclusion criteria were menopause (defined as a combination of folliclestimulating hormone [FSH] levels above $35 \mathrm{IU} / \mathrm{L}$ and last menstrual period at least 1 year before the beginning of the study), age between 45 and 65 years, and no use of hormone therapy for at least 3 months before the enrollment. Exclusion criteria were prior diagnosis of CVD, current smoking, or a diagnosis of diabetes. The local Research Ethics Committee from Hospital de Clinicas de
Porto Alegre approved the study, and each participant provided written informed consent.

\section{Study protocol}

Anthropometric measurements included body weight, height, and body mass index (BMI, calculated as the latest measured weight in kilograms divided by the height in meters squared). Blood pressure was measured twice with a 10-min interval using an automatic blood pressure monitor (HEM-742INT; Omron, Rio de Janeiro, Brazil). Participants were in a seated position, with feet on the floor and the arm supported at heart level.

$\mathrm{CV}$ risk was estimated by using the Framingham General Cardiovascular Risk Score (10-year risk) (FRS), which was determined, using lipids, through the online interactive risk score calculator available on the Framingham Heart Study website [32]. Participants were stratified into two groups according to FRS: $<10 \%(n=69)$, representing a low risk group; and $\geq 10 \%$, representing an intermediate/high risk group $(n=21)$.

\section{Global DNA methylation assays}

Venous blood samples were collected. Genomic DNA was extracted from peripheral leukocytes using the technique described by Miller et al. [33]. The extracted peripheral leukocyte DNA was quantified using a NanoDrop ${ }^{\mathrm{TM}} 1000$ Spectrophotometer (Thermo Scientific, Wilmington, DE).

Global DNA methylation levels were obtained with an ELISA-based commercial kit (MDQ1, Imprint ${ }^{\circ}$ Methylated DNA Quantification Kit, Sigma Aldrich, St. Louis, MO, USA). The MDQ1 kit is a high-throughput molecular biology kit that uses a 96-well plate format to provide accurate differential global DNA methylation. The samples were incubated with capture and detection antibodies and absorbance was read at 450 nanometers. Quantification of global DNA methylation was obtained from calculating the amount of methylated cytosines in the samples $(5 \mathrm{mC})$ relative to global cytidine $(5 \mathrm{mC}+\mathrm{dC})$ in methylated control DNA (50 ng/ $\mu \mathrm{L})$. For each sample, methylation analysis was performed in duplicate (200 ng DNA each), and the average of these measurements is reported. The intra and interassay coefficient of variation (CV) were $<10 \%$.

\section{Biochemical and hormone assays}

Serum estradiol, insulin, high-sensitive C-reactive protein (hs-CRP), glucose and lipid profile (triglycerides, total cholesterol, and high-density lipoprotein [HDL] cholesterol) were determined in a 12-hour fasting blood sample collected between 8 and 10 a.m. and assessed by colorimetric-enzymatic methods (Siemens Advia 1800 System, Deerfield, USA) with a coefficient of variation $(\mathrm{CV})<3.4 \%$. Low-density lipoprotein (LDL) cholesterol was estimated indirectly using the Friedewald formula 
[34]. Serum insulin levels were measured using CLIA (Siemens Centaur XP, Deerfield, USA), with a sensitivity of $0.20 \mu \mathrm{IU} / \mathrm{mL}$ and intra- and interassay $\mathrm{CV}$ of 2.0 and $4.3 \%$ respectively. Homeostatic model assessment index (HOMA) was calculated by multiplying insulin $(\mu \mathrm{IU} / \mathrm{mL})$ by glucose $(\mathrm{mmol} / \mathrm{L})$ and dividing this product by 22.5 , as previously described [35]. Estradiol was measured by ECLIA (Roche Diagnostics, Mannheim, Germany), with an assay sensitivity of $5.0 \mathrm{pg} / \mathrm{mL}$ and intra- and interassay CVs of 5.7 and $6.4 \%$ respectively. Serum hs-CRP was assayed by nephelometric method (Dade Behring Marburg, Marburg, Germany). Sensitivity was $0.17 \mathrm{mg} / \mathrm{L}$ and intra and interassay CVs were 4.4 and $5.7 \%$ respectively. For data analysis, individual results below the sensitivity of the assay were considered as equal to $0.17 \mathrm{mg} / \mathrm{L}$.

\section{Statistical analysis}

Descriptive data were expressed as mean \pm standard deviation (SD) or median and interquartile range (IQR) (25-75 \%). Variables with non-parametric distribution were transformed into logarithms for calculation, and converted back to their original form for data presentation. Student's $t$ test was used for comparisons between group means. Global DNA methylation analyses were adjusted for time since menopause (linear regression). All analyses were performed using the Statistical Package for the Social Sciences (SPSS) version 20 (SPSS Inc., Chicago, IL, USA). Findings were deemed significant at $p<0.05$.

\section{Results}

Considering the overall sample, mean age was $55.5 \pm$ 4.9 years and mean BMI was $27.2 \pm 4.6 \mathrm{~kg} / \mathrm{m}^{2}$. Table 1 shows anthropometric and metabolic data for the entire group and for each FRS group. Patients with FRS $\geq 10 \%$ were older than the group with FRS $<10 \%$. Time since menopause, blood pressure, total cholesterol, and LDL-c levels were also higher in the FRS $\geq 10$ \% group in comparison with the FRS $<10 \%$ group. Conversely, the two groups had similar BMI, estradiol, triglycerides, HDL-c, HOMAIR, glucose and high-sensitive C-reactive protein levels.

Global DNA methylation results (\% $5 \mathrm{mC}$ ) for the overall sample and FRS groups are also presented in Table 1. The group with FRS $\geq 10 \%$ had lower global methylation levels compared with the FRS $<10 \%$ group (23.9 \% [20.6-29.1] vs. $28.8 \%$ [24.3-39.6], $p=0.02$ ), even after adjustment for time since menopause $(p=0.02)$ (Fig. 1).

\section{Discussion}

In this cross-sectional study with postmenopausal women, we found that global methylation levels were lower in participants in the intermediate/high CV risk group (FRS $\geq 10 \%$ ) as compared to those with low CV risk (FRS $<10 \%$ ). To the best of our knowledge, this is the first study to specifically examine the association between global DNA methylation and CV risk in postmenopausal women with no clinical disease.

Age and time since menopause are well-known CV risk factors [36, 37]. Indeed, decreased estrogen levels have been implicated in the progression of atherosclerosis after menopause [36, 37]. In addition, lifestyle is also linked to $\mathrm{CV}$ risk in the menopausal transition and postmenopausal period $[38,39]$. However, in the present study, similar low circulating estrogen levels were observed in the groups with FRS $<10$ and FRS $\geq 10 \%$ - despite the higher age and time since menopause in the latter group. Also, the differences between these groups in methylation levels persisted after adjustment for time since menopause. This observation underscores the relevance of epigenetic mechanisms along with life style and estrogenicity for CV risk, especially in postmenopausal women.

Previous studies have associated global DNA methylation changes with clinical and subclinical CVD risk components, such as atherosclerosis, hypertension, and coronary artery disease, in different populations [26-30]. Global DNA methylation has been found to be lower in hypertensive subjects than in normotensive controls, with $5 \mathrm{mC}$ levels correlating with stage of hypertension [40]. Moreover, a study analyzing long interspersed nucleotide elements (LINE-1) as a surrogate marker of global DNA methylation status in blood cells found an association of lower methylation levels with cardiovascular events, such as stroke and ischemic heart disease in elderly men [41]. Another study has recently reported that lower global DNA methylation was also associated with stroke [26]. Taken together, the results of the present study and those produced by other investigators suggest that global methylation status may be regarded as a marker of CV risk in postmenopausal women. Further studies are needed in order to better understand the mechanisms underlying this association.

Animal studies have also shown that DNA methylation plays a critical role in the development of atherosclerosis and CVD. In a murine model, the absence of DNMT1 was related to increased expression of inflammatory mediators [42]. Additionally, genetically atherosclerosisprone mice lacking apolipoprotein $\mathrm{E}$ showed changes in DNA methylation in peripheral blood leukocytes, which contributed to dysregulation of inflammation and promotion of atherosclerosis [43]. A modest but significant global hypomethylation status was also observed in aortic samples, and this condition preceded any histological evidence of atherosclerosis [43].

While the predominant form of methylation occurs at cytosines that are adjacent to guanine, separated by only one phosphate $(\mathrm{CpG})$, in the present study we also assessed $\mathrm{CpH}$ sites. In this situation, a methyl group is added to a cytosine that is upstream to an adenine, thymine, or another cytosine. In fact, previous studies have 
Table 1 Distribution of anthropometric and metabolic variables according to Framingham Risk Score

\begin{tabular}{|c|c|c|c|c|}
\hline Characteristic & Overall $(n=90)$ & FRS $<10 \%(n=69)$ & FRS $\geq 10 \%(n=21)$ & $p^{*}$ \\
\hline Age (years) & $55.5 \pm 4.9$ & $54.4 \pm 4.4$ & $59.1 \pm 4.6$ & 0.001 \\
\hline Time since menopause (years) & $5.8(3.0-10.0)$ & $5.0(2.5-9.0)$ & $10(4.0-13.2)$ & 0.004 \\
\hline BMI $\left(\mathrm{kg} / \mathrm{m}^{2}\right)$ & $27.2 \pm 4.6$ & $26.9 \pm 4.7$ & $28.0 \pm 4.3$ & 0.34 \\
\hline $\mathrm{SBP}(\mathrm{mmHg})$ & $127.5 \pm 17.3$ & $120.6 \pm 11.2$ & $150.8 \pm 13.5$ & 0.001 \\
\hline $\mathrm{DBP}(\mathrm{mmHg})$ & $78.9 \pm 10.1$ & $76.4 \pm 9.2$ & $87.3 \pm 8.6$ & 0.001 \\
\hline Fasting glucose (mg/dL) & $93.3 \pm 8.9$ & $93.6 \pm 9.1$ & $92.1 \pm 8.0$ & 0.47 \\
\hline Total cholesterol (mg/dL) & $216.5 \pm 33.8$ & $212.3 \pm 32.7$ & $231.0 \pm 34.4$ & 0.02 \\
\hline LDL-c (mg/dL) & $139.2 \pm 28.8$ & $135.3 \pm 27.3$ & $152.8 \pm 30.2$ & 0.01 \\
\hline $\mathrm{HDL}-\mathrm{c}(\mathrm{mg} / \mathrm{dL})$ & $53.9 \pm 12.7$ & $53.4 \pm 13.2$ & $55.5 \pm 10.7$ & 0.49 \\
\hline Triglycerides (mg/dL) & $93.0(72.0-139.7)$ & $91.0(70.0-141.2)$ & $105.5(75.2-141.0)$ & 0.87 \\
\hline HOMA-IR & $1.92(1.30-3.19)$ & $1.9(1.3-3.2)$ & $1.9(1.2-2.9)$ & 0.68 \\
\hline hs-CRP (mg/L) & $1.30(0.37-3.66)$ & $1.0(0.3-3.3)$ & $2.5(0.5-5.5)$ & 0.27 \\
\hline Estradiol (pg/mL) & $20.4(12.0-31.0)$ & $20.3(12.0-31.5)$ & $22.4(13.4-30.8)$ & 0.92 \\
\hline Global DNA methylation (\% 5 mC) & $26.5(23.6-36.9)$ & & & \\
\hline
\end{tabular}

Data are given as mean \pm standard deviation or median and interquartile range (25-75 \%). ${ }^{*} p$ by Student's $t$ test, FRS $<10 \%$ vs. FRS $\geq 10 \%$

$B M I$ body mass index, DBP diastolic blood pressure, FRS Framingham Risk Score, $H D L$-c high-density lipoprotein cholesterol, $H O M A-I R$ homeostasis model assessment -

insulin resistance, $h s$ - $C R P$ high-sensitive $C$-reactive protein, $L D L-c$ low-density lipoprotein cholesterol, SBP systolic blood pressure

shown that $\mathrm{CpH}$ methylation might be more common than previously appreciated $[44,45]$. Moreover, $\mathrm{CpG}$ and $\mathrm{CpH}$ methylation may have distinct functions, with $\mathrm{CpG}$ sites methylated symmetrically and $\mathrm{CpH}$ sites methylated in strand-specific fashion in introns and repetitive DNA elements (SINE and LINE) [46].

Finally, we showed similar quantification of $5 \mathrm{mC}$ when compared with previous studies analyzing global DNA methylation in leukocytes in different populations $[47,48]$. There is a wide range of methods to evaluate methylation status. Recent advances in sequencing and microarray technology make it possible to map DNA methylation genome-wide at good resolution and in a large number of samples $[49,50]$. However, these technologies may be very expensive and data processing and biological interpretation are still challenging [16]. Thus, measuring methylation levels using an ELISA assay, a well-known, simple, and reproducible technique can be helpful to guide future studies with whole-genome bisulfite sequencing.

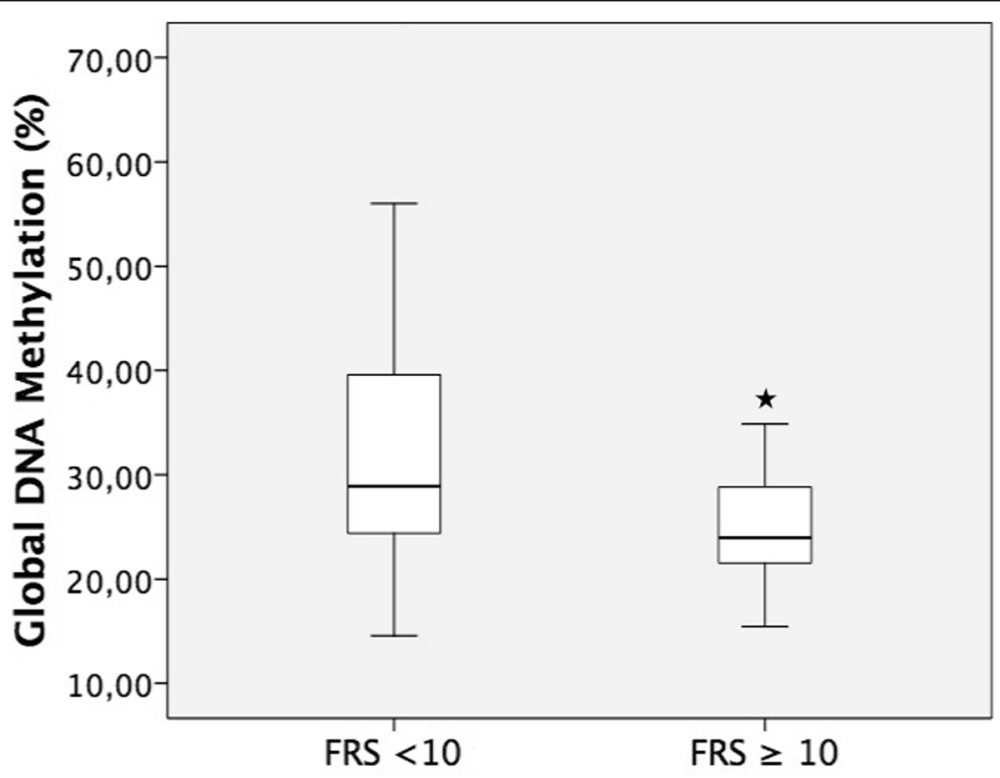

Fig. 1 Percent global DNA methylation in FRS $<10$ and FRS $\geq 10 \%$ groups. Values are expressed as median and $25-75 \%$ IQR (lower and upper limit of the box); maximum and minimum values are shown by the limits of vertical lines. ${ }^{*} p=0.02$ by Student's $t$ test 
Limitations of the present study include the crosssectional design, which precludes conclusions regarding the direction of cause and effect. Another limitation is the relatively small sample size, which may have limited the statistical power for some analyses, such as those related to the influence of age on the observed findings. However, the effect sizes observed in our sample are similar to those reported in other populations with the same age and with established cardiovascular disease.

\section{Conclusion}

In conclusion, our results indicate that lower global DNA methylation is associated with higher CV risk in postmenopausal women with no clinical evidence of disease. This finding should be further investigated in large-scale epidemiological studies to assess epigenetic mechanisms of CVD in this population.

\section{Abbreviations}

BMI: Body mass index; CV: Cardiovascular; CVD: Cardiovascular disease; DBP: Diastolic blood pressure; DNMTs: DNA methyltransferases; FRS: Framingham risk score; FSH: Follicle-stimulating hormone; HDL-c: High-density lipoprotein cholesterol; HOMA-IR: Homeostasis model assessment - insulin resistance; hs-CRP: High-sensitive C-reactive protein; IQR: Interquartile range; LDL-C: Low-density lipoprotein cholesterol; LINE-1: Long interspersed nucleotide elements; SBP: Systolic blood pressure; SD: Standard deviation

\section{Acknowledgements}

We thank Thais Rasia da Silva and Roberta Frantz for their support with clinical data collection

\section{Funding}

This work was supported by the Brazilian National Institute of Hormones and Women's Health/Conselho Nacional de Desenvolvimento Científico e Tecnológico (CNPq INCT 573747/2008-3). The funding sources were not involved in study design; in the collection, analysis and interpretation of data; in the writing of the report; or in the decision to submit the paper for publication.

\section{Availability of data and materia}

All data analyzed during this study are included in this published article.

\section{Authors' contributions}

RBR participated in the conception and design of the study, carried out the DNA methylation assays, was involved with data analysis and drafted the manuscript; VF was involved with data analysis and drafted the manuscript; SBL participated in the DNA methylation assays, was involved with data analysis and helped to draft the manuscript; MAM was involved in the data collection and analysis; PMS conceived the study, participated in its design and coordination, was involved in the data analysis and helped to draft the manuscript. All authors read and approved the final manuscript.

\section{Competing interests}

The authors declare that they have no competing interests.

\section{Consent for publication}

Not applicable.

\section{Ethics approval and consent to participate}

The local Research and Ethics Committee from Hospital de Clinicas de Porto Alegre approved the study (GPPG 100317) and each participant provided written informed consent.

\section{Author details}

${ }^{1}$ Gynecological Endocrinology Unit, Division of Endocrinology, Hospital de Clínicas de Porto Alegre, Rua Ramiro Barcelos, 2350, Porto Alegre, RS 90035-003, Brazil. ²Department of Diagnostic Methods, Universidade Federal de Ciências da Saúde de Porto Alegre, Rua Sarmento Leite, 245, Porto Alegre, RS 90050-170, Brazil. 'Laboratory of Molecular Endocrinology, Department of Physiology, Universidade Federal do Rio Grande do Sul, Rua Ramiro Barcelos, 2350, Porto Alegre, RS 90035-003, Brazil. ${ }^{4}$ Present addresses: Institute of Cardiology of Rio Grande do Sul, Cardiology University Foundation, Avenida Princesa Isabel, 395, Porto Alegre, RS 90040-371, Brazil. ' Unisinos University, Av. Unisinos, 950, São Leopoldo, RS 93022-000, Brazil.

Received: 6 December 2015 Accepted: 5 October 2016

Published online: 10 October 2016

\section{References}

1. Colditz GA, Willett WC, Stampfer MJ, et al. Menopause and the risk of coronary heart disease in women. N Engl J Med. 1987;316:1105-10.

2. Vaidya $D$, Becker DM, Bittner $V$, et al. Ageing, menopause, and ischaemic heart disease mortality in England, Wales, and the United States: modelling study of national mortality data. BMJ. 2011;343:d5170.

3. Davis PH, Dawson JD, Riley WA, et al. Carotid intimal-medial thickness is related to cardiovascular risk factors measured from childhood through middle age: the Muscatine study. Circulation. 2001;104:2815-9.

4. Koskinen J, Kahonen M, Viikari JS, et al. Conventional cardiovascular risk factors and metabolic syndrome in predicting carotid intima-media thickness progression in young adults: the cardiovascular risk in young Finns study. Circulation. 2009:120:229-36.

5. Pappa T, Alevizaki M. Endogenous sex steroids and cardio- and cerebro-vascular disease in the postmenopausal period. Eur J Endocrinol. 2012;167:145-56.

6. Stamatelopoulos KS, Armeni E, Georgiopoulos G, et al. Recently postmenopausal women have the same prevalence of subclinical carotid atherosclerosis as age and traditional risk factor matched men. Atherosclerosis. 2012;221:508-13.

7. El Khoudary SR, Wildman RP, Matthews K, et al. Progression rates of carotid intima-media thickness and adventitial diameter during the menopausal transition. Menopause. 2013:20:8-14.

8. Lambrinoudaki I, Armeni E, Georgiopoulos G, et al. Subclinical atherosclerosis in menopausal women with low to medium calculated cardiovascular risk. Int J Cardiol. 2013;164:70-6.

9. Sayols-Baixeras S, Lluis-Ganella C, Lucas G, et al. Pathogenesis of coronary artery disease: focus on genetic risk factors and identification of genetic variants. Appl Clin Genet. 2014:7:15-32.

10. Joseph PG, Pare G, Anand SS. Exploring gene-environment relationships in cardiovascular disease. Can J Cardiol. 2013;29:37-45.

11. Dawber TR, Meadors GF, Moore Jr FE. Epidemiological approaches to heart disease: the Framingham Study. Am J Public Health Nations Health. 1951:41:279-81

12. D'Agostino Sr RB, Vasan RS, Pencina MJ, et al. General cardiovascular risk profile for use in primary care: the Framingham Heart Study. Circulation. 2008;117:743-53.

13. Whitfield JB. Genetic insights into cardiometabolic risk factors. Clin Biochem Rev. 2014;35:15-36.

14. Turgeon PJ, Sukumar AN, Marsden PA. Epigenetics of cardiovascular disease - a new "Beat" in coronary artery disease. Med Epigenet. 2014;2:37-52.

15. Manolio TA, Collins FS, Cox NJ, et al. Finding the missing heritability of complex diseases. Nature. 2009;461:747-53.

16. Ladd-Acosta C, Fallin MD. The role of epigenetics in genetic and environmental epidemiology. Epigenomics. 2016;8(2):271-83.

17. Waddington $\mathrm{CH}$. Canalization of development and genetic assimilation of acquired characters. Nature. 1959;183:1654-5.

18. Heyn H, Moran S, Hernando-Herraez I, et al. DNA methylation contributes to natural human variation. Genome Res. 2013:23:1363-72.

19. Moore LD, Le T, Fan G. DNA methylation and its basic function. Neuropsychopharmacology. 2013;38:23-38.

20. Whayne TF. Epigenetics in the development, modification, and prevention of cardiovascular disease. Mol Biol Rep. 2015:42:765-76.

21. Barlow DP, Bartolomei MS. Genomic imprinting in mammals. Cold Spring Harb Perspect Biol. 2014;6:a018382. [https://www.ncbi.nlm.nih.gov/pmc/ articles/PMC3941233/pdf/cshperspect-EPI-a018382.pdf]

22. Jin B, Robertson KD. DNA methyltransferases, DNA damage repair, and cancer. Adv Exp Med Biol. 2013;754:3-29.

23. Bestor TH. The DNA methyltransferases of mammals. Hum Mol Genet. 2000:9:2395-402. 
24. Guerrero-Preston R. Global epigenetic screening technologies: a novel too to address cancer health disparities in high-risk population groups. $\mathrm{P} R$ Health Sci J. 2008;27:350-6.

25. Guerrero-Preston R, Herbstman J, Goldman LR. Epigenomic biomonitors: global DNA hypomethylation as a biodosimeter of life-long environmenta exposures. Epigenomics. 2011;3:1-5.

26. Soriano-Tarraga C, Jimenez-Conde J, Giralt-Steinhauer E, et al. Global DNA methylation of ischemic stroke subtypes. PLoS One. 2014;9:e96543.

27. Wilson AS, Power BE, Molloy PL. DNA hypomethylation and human diseases. Biochim Biophys Acta. 2007;1775:138-62.

28. Castro R, Rivera I, Struys EA, et al. Increased homocysteine and Sadenosylhomocysteine concentrations and DNA hypomethylation in vascular disease. Clin Chem. 2003;49:1292-6.

29. Nanayakkara PW, Kiefte-de Jong JC, Stehouwer CD, et al. Association between global leukocyte DNA methylation, renal function, carotid intimamedia thickness and plasma homocysteine in patients with stage 2-4 chronic kidney disease. Nephrol Dial Transplant. 2008;23:2586-92.

30. Ordovas JM, Smith CE. Epigenetics and cardiovascular disease. Nat Rev Cardiol. 2010;7:510-9.

31. Maturana MA, Franz RF, Metzdorf M, et al. Subclinical cardiovascular disease in postmenopausal women with low/medium cardiovascular risk by the Framingham risk score. Maturitas. 2015;81:311-6.

32. Framingham HS. Secondary. 2015. http://www.framinghamheartstudy.org/ riskfunctions/cardiovascular-disease/10-year-risk.php.

33. Miller SA, Dykes DD, Polesky HF. A simple salting out procedure for extracting DNA from human nucleated cells. Nucleic Acids Res. 1988;16:1215.

34. Friedewald WT, Levy RI, Fredrickson DS. Estimation of the concentration of low-density lipoprotein cholesterol in plasma, without use of the preparative ultracentrifuge. Clin Chem. 1972;18:499-502.

35. Matthews DR, Hosker JP, Rudenski AS, et al. Homeostasis model assessment: insulin resistance and beta-cell function from fasting plasma glucose and insulin concentrations in man. Diabetologia. 1985;28:412-9.

36. Bittner V. Menopause, age, and cardiovascular risk: a complex relationship. J Am Coll Cardiol. 2009:54:2374-5.

37. Coylewright M, Reckelhoff JF, Ouyang P. Menopause and hypertension: an age-old debate. Hypertension. 2008;51:952-9.

38. Silva TR, Alves BC, Maturana MA, et al. Healthier dietary pattern and lower risk of metabolic syndrome in physically active postmenopausal women. J Am Coll Nutr. 2013;32:287-95.

39. Colpani V, Oppermann K, Spritzer PM. Association between habitual physical activity and lower cardiovascular risk in premenopausal, perimenopausal, and postmenopausal women: a population-based study. Menopause. 2013;20:525-31.

40. Smolarek I, Wyszko E, Barciszewska AM, et al. Global DNA methylation changes in blood of patients with essential hypertension. Med Sci Monit. 2010;16:CR149-55

41. Baccarelli A, Wright $R$, Bollati $V$, et al. Ischemic heart disease and stroke in relation to blood DNA methylation. Epidemiology. 2010;21:819-28.

42. Makar KW, Wilson CB. DNA methylation is a nonredundant repressor of the Th2 effector program. J Immunol. 2004;173:4402-6.

43. Lund $G$, Andersson L, Lauria $M$, et al. DNA methylation polymorphisms precede any histological sign of atherosclerosis in mice lacking apolipoprotein E. J Biol Chem. 2004;279:29147-54.

44. Lister R, Pelizzola M, Dowen RH, et al. Human DNA methylomes at base resolution show widespread epigenomic differences. Nature. 2009:462:315-22.

45. Ramsahoye BH, Biniszkiewicz D, Lyko F, et al. Non-CpG methylation is prevalent in embryonic stem cells and may be mediated by DNA methyltransferase 3a. Proc Natl Acad Sci U S A. 2000;97:5237-42.

46. Guo W, Chung WY, Qian M, et al. Characterizing the strand-specific distribution of non-CpG methylation in human pluripotent cells. Nucleic Acids Res. 2014;42:3009-16.

47. Tellez-Plaza M, Tang WY, Shang Y, et al. Association of global DNA methylation and global DNA hydroxymethylation with metals and other exposures in human blood DNA samples. Environ Health Perspect. 2014;122:946-54.

48. Guerrero-Preston R, Goldman LR, Brebi-Mieville P, et al. Global DNA hypomethylation is associated with in utero exposure to cotinine and perfluorinated alkyl compounds. Epigenetics. 2010;5:539-46.

49. Laird PW. Principles and challenges of genomewide DNA methylation analysis. Nat Rev Genet. 2010;11:191-203.

50. Bock C. Analysing and interpreting DNA methylation data. Nat Rev Genet. 2012;13:705-19.

\section{Submit your next manuscript to BioMed Central and we will help you at every step:}

- We accept pre-submission inquiries

- Our selector tool helps you to find the most relevant journal

- We provide round the clock customer support

- Convenient online submission

- Thorough peer review

- Inclusion in PubMed and all major indexing services

- Maximum visibility for your research

Submit your manuscript at www.biomedcentral.com/submit
) Biomed Central 